\title{
Pairing and density-wave phases in Boson-Fermion mixtures at fixed filling
}

\author{
F. D. Klironomo: and S.-W. Tsa: \\ Physics Department, University of California, Riverside, CA 92521
}

(Dated: July 7, 2018)

\begin{abstract}
We study a mixture of fermionic and bosonic cold atoms on a two-dimensional optical lattice, where the fermions are prepared in two hyperfine (isospin) states and the bosons have Bose-Einstein condensed (BEC). The coupling between the fermionic atoms and the bosonic fluctuations of the BEC has similarities with the electron-phonon coupling in crystals. We study the phase diagram for this system at fixed fermion density of one per site (half-filling). We find that tuning of the lattice parameters and interaction strengths (for fermion-fermion, fermion-boson and boson-boson interactions) drives the system to undergo antiferromagnetic ordering, s-wave and d-wave pairing superconductivity or a charge density wave phase. We use functional renormalization group analysis where retardation effects are fully taken into account by keeping the frequency dependence of the interaction vertices and self-energies. We calculate response functions and also provide estimates of the energy gap associated with the dominant order, and how it depends on different parameters of the problem.
\end{abstract}

PACS numbers: 03.75.Mn,05.10.Cc,71.10.Fd,71.10.Hf

Recent advances in manipulating ultracold atoms in optical lattices have produced remarkable results spanning a variety of modern condensed matter physics phenomena such as the realization of the Mott-insulator transition [1], fermionic superfluidity [2], the realization of the Tonks-Girardeau gas 3], and the BerezinskiiKosterlitz-Thouless transition [4], just to name a few indicative ones. The experimental control over a wide range of parameters associated with these type of systems renders them a favorite physics playground both for experimentalists and theorists alike. In particular, a boson-fermion mixture (BFM) system can provide a fascinating testing ground for a large section of theoretical physics [5] furthering our understanding of condensed matter phenomena.

Previous work has explored part of the vast and rich phase space associated with this two-species system by employing mean-field or variational type of approaches and allowing for fluctuating occupation numbers for both fermions and bosons [6, 7, [8, 9, 10]. As expected, these approaches provide a good general picture of the rich phasespace but are unable to capture the delicate interplay and competition of correlation effects in a quantitative manner which can be a useful reference for experimentalists. A more recent work, based on our methodology of analysis [11], was the first approach to study this type of system beyond mean-field limitations but (as in all previous studies) was applied only for the regime $v_{F} \ll v_{s}$, where retardation effects between the fermionic dynamics dictated by the Fermi velocity $v_{F}$ and the bosonic dynamics dictated by the sound velocity $v_{s}$ are not important. It is very important to investigate the physics beyond this regime since $v_{F} \geq v_{s}$ is experimentally accessible and in fact, retardation can play a crucial role in the interplay of orders. The above considerations have prompted us to focus our attention on this type of system and complement on the physical understanding of it by applying our theoretical apparatus originally developed for the electron-phonon type of system[12].

The framework under which we work is based on functional renormalization group (fRG) analysis for twodimensional fermions 13] in the presence of phonons when full retardation is taken into consideration 14. This approach has the important advantage of including all competing fermionic processes under a given (one-loop) accuracy approximation in an unbiased way, so that all possible orders are studied on an equal basis, contrary to mean field theory for example which always presupposes a manifested order. The only necessary ingredient that is required for this study to work is the bosonic system to be in the Bose-Einstein condensation phase (BEC) which leads to an electron-phonon type of fermion-boson interaction (linear to first order in the bosonic field) [11]. This allows us to integrate the bosons out and build an effective fermionic theory, and consequently apply the fRG analysis to investigate all possible orders of interest the fermions can undergo, while taking retardation effects fully into account. What is interesting to realize is that our study can be generalized for any type of a two-component itinerant fermionic system on a square lattice in the presence of a gapless collective mode that renormalizes attractively the fermionic interaction. All of our results will remain valid within that context.

The standard model used to study itinerant fermions and bosons on a lattice is the Hubbard model. For a typical BFM system consisting of neutral atoms $\left({ }^{40} \mathrm{~K}\right.$ for fermions and ${ }^{87} \mathrm{Rb}$ for bosons) that interact through short-range van der Waals forces a good approximation consists only of hardcore (onsite) intra-species (fermionfermion $U_{f f}$, boson-boson $U_{b b}$ ) repulsive interactions and inter-species (fermion-boson $U_{f b}(\boldsymbol{q})$ ) repulsive or attractive interactions. Additionally, the presence of a twocomponent isospin quantum number among fermions relaxes Pauli's exclusion principle limitations for s-wave 
type of scattering events (lowest in energy) and opens up a large and rich phase space previously inaccessible for "spinless" fermionic atoms. This type of two-component fermionic system in the presence of a collective mode can effectively map and simulate a large variety of correlated electronic systems in condensed matter physics [5]. The role of isospin in this case is played by different total angular momentum projection states for the ${ }^{40} \mathrm{~K}$ atoms, which can additionally be exploited for Feshbach resonance type of tuning of the inter-species scattering strength $U_{f b}[15]$.

The Hamiltonian for this system of itinerant fermions and bosons on a two-dimensional square lattice has the form

$$
\begin{aligned}
& H=\sum_{\boldsymbol{k}, \sigma} \xi_{\boldsymbol{k}}^{f} f_{\boldsymbol{k}, \sigma}^{\dagger} f_{\boldsymbol{k}, \sigma}+\sum_{\boldsymbol{q}} \xi_{\boldsymbol{q}}^{b} B_{\boldsymbol{q}}^{\dagger} B_{\boldsymbol{q}} \\
& +\frac{1}{2 V} \sum_{\boldsymbol{k}, \boldsymbol{k}^{\prime}, \boldsymbol{q}} U_{b b} B_{\boldsymbol{k}+\boldsymbol{q}}^{\dagger} B_{\boldsymbol{k}} B_{\boldsymbol{k}^{\prime}-\boldsymbol{q}}^{\dagger} B_{\boldsymbol{k}^{\prime}} \\
& +\frac{1}{V} \sum_{\boldsymbol{k}, \boldsymbol{k}^{\prime}, \boldsymbol{q}, \sigma} U_{f b}(\boldsymbol{q}) f_{\boldsymbol{k}+\boldsymbol{q}, \sigma}^{\dagger} f_{\boldsymbol{k}, \sigma} B_{\boldsymbol{k}^{\prime}-\boldsymbol{q}}^{\dagger} B_{\boldsymbol{k}^{\prime}} \\
& +\frac{1}{V} \sum_{\boldsymbol{k}, \boldsymbol{k}^{\prime}, \boldsymbol{q}} U_{f f} f_{\boldsymbol{k}+\boldsymbol{q}, \uparrow}^{\dagger} f_{\boldsymbol{k}, \uparrow} f_{\boldsymbol{k}^{\prime}-\boldsymbol{q}, \downarrow}^{\dagger} f_{\boldsymbol{k}^{\prime}, \downarrow}
\end{aligned}
$$

where $f_{\boldsymbol{k}, \sigma}, B_{\boldsymbol{q}}$ are the fermion, boson operators respectively, $\xi_{\boldsymbol{k}}^{f, b}=-2 t_{f, b}\left(\cos k_{x}+\cos k_{y}\right)-\mu_{f, b}$ are the corresponding dispersion relations dictated by the square lattice symmetry, with $t_{f, b}$ being the overlap integrals and $\mu_{f, b}$ the chemical potentials for the fermions and bosons respectively.

For the purpose of this study we will focus our attention at half-filling for the fermions $\left(\mu_{f}=0\right)$ where the pairing-related phases ( $\mathrm{s}$,d-wave) superconductivity (sSC, dSC) can compete with the nesting-related phases like charge density wave (CDW) and antiferromagnetism (SDW), and a rich landscape of phase space becomes available for the fermionic system to explore 14]. This amounts to fermion numbers in the range $10^{3}-10^{4}$ for typical optical lattice parameters $a=400 \mathrm{~nm}$ on $2 \mathrm{D}$ optical harmonic traps of frequency $\omega=2 \pi \times 30 \mathrm{~Hz}$, which is well within experimental capabilities. Additionally, at half-filling besides the presence of nesting on the Fermi surface there are van Hove singularities as well that can enhance the interplay and competition among all orders. Our assumption of BEC for the bosons allows us to expand the bosonic operators in Eq. (1) according to $B_{\boldsymbol{q}}=\sqrt{N_{0}} \delta_{\boldsymbol{q}, 0}+B_{\boldsymbol{q}}$, where the creation (B) or destruction $\left(B_{\boldsymbol{q}}\right)$ of quasiparticles follows from fluctuations $\left(B_{\mathbf{0}}\right)$ of the macroscopic number $N_{0}$ of condensate atoms. Consequently we can perform the usual Bogoliubov transformation and linearize the inter-species interaction introducing the gapless collective mode

$$
\omega_{\boldsymbol{q}}=\sqrt{\varepsilon_{\boldsymbol{q}}\left(\varepsilon_{\boldsymbol{q}}+2 n_{b} U_{b b}\right)},
$$

where $\varepsilon_{\boldsymbol{q}}=\xi_{\boldsymbol{q}, \mu_{b}=-4 t_{b}}^{b}$ is the free-boson dispersion relation (chemical potential exactly at the non-interacting ground state), and $n_{b}=N_{0} / V$ is the BEC density which for all practical purposes can be considered as the bosonic filling factor on the lattice.

This is the fixed point of our theory as far as the stability of the bosonic system in the presence of fermions is concerned. It has been experimentally shown [16, 17, 18] that the BEC can be stable in the presence of fermionic atoms provided the two-species numbers do not exceed $10^{5}$ atoms in the optical trap in the absence of an optical lattice. The presence of an optical lattice will stabilize the system against phase separation or collapse to even higher densities, since both processes rely on the interspecies interaction $U_{f b}$ dominating over the kinetic energy scales associated with the weakly confined atoms, and three-body recombination processes becoming relevant as well. The lattice will introduce experimentally tunable kinetic energy scales associated with the hopping of atoms from lattice point to lattice point $\left(t_{f, b}\right)$ competing with $U_{f b}$ and consequently stabilizing the system.

Dynamical fluctuations of the BFM around this fixed point can be modeled in the Matsubara representation. After the bosonic fields associated with fluctuations of the BEC are integrated out 11, 12 we arrive at the fully retarded effective fermionic interaction given by

$$
\widetilde{U}_{f f}\left(k_{1}, k_{2}, k_{3}\right)=U_{f f}-\frac{U_{f b}^{2} / U_{b b}}{1+4 \xi^{2}-2 \xi^{2}\left(\cos \left(\boldsymbol{k}_{1}-\boldsymbol{k}_{3}\right)_{x}+\cos \left(\boldsymbol{k}_{1}-\boldsymbol{k}_{3}\right)_{y}\right)} \frac{\omega_{\boldsymbol{k}_{1}-\boldsymbol{k}_{3}}^{2}}{\left(\omega_{1}-\omega_{3}\right)^{2}+\omega_{\boldsymbol{k}_{1}-\boldsymbol{k}_{3}}^{2}},
$$

where $k_{i} \equiv\left(i \omega_{n_{i}}, \boldsymbol{k}_{i}\right), \omega_{n_{i}}$ are the fermionic Matsubara frequencies, and $\xi=\sqrt{t_{b} / 2 n_{b} U_{b b}}$ is the healing length of the BEC. As we see, fluctuations of the BEC renormalize $U_{f f}$ with an attractive and frequency-dependent part which is not affected by the attractive or repulsive nature of the inter-species interaction $U_{f b}[8]$. The importance of retardation is determined by Eq. (2) which in the longwavelength limit defines the acoustic velocity $v_{s}=\sqrt{2 t_{b} U_{b b} n_{b}}$. For fermionic velocities $\left(v_{F} \simeq 2 t_{f}\right)$ much smaller than $v_{s}$ one recovers the mean-field limit 


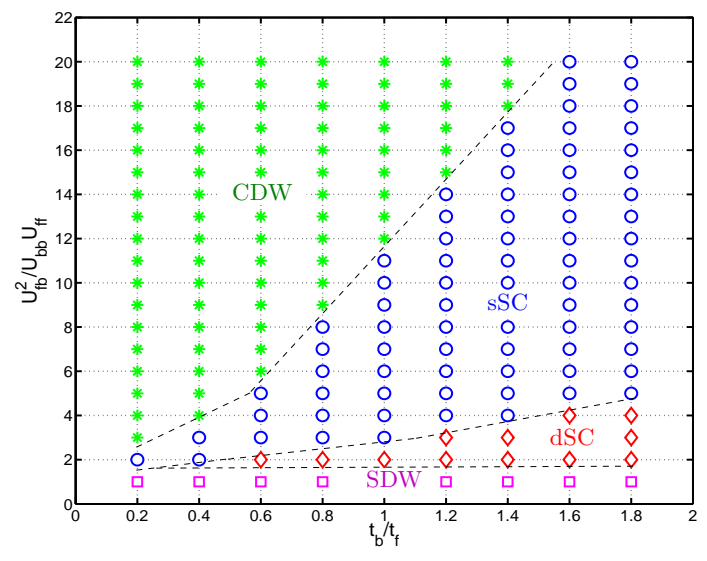

FIG. 1: (Color online) Phase diagram for $U_{f f}=0.4 t_{f}, U_{b b}=$ $0.8 t_{f}$, and $n_{b}=2.5$. Blue circles indicate sSC, red rhombuses indicate dSC, magenta squares SDW, and green stars CDW type of ordering. Dashed lines are guides to the eye.

for $\widetilde{U}_{f f}[8]$. On the other hand, retardation dominates for $v_{s} \leq v_{F}$ and the competition of phases is enhanced. This regime can be achieved experimentally since there are two tunable parameters $\left(t_{b}, U_{b b}\right)$ that can be adjusted in situ for a given system configuration $\left(n_{b}\right)$. The healing length $\xi$ plays an important role in the competition of phases since it defines the length-scale over which bosonic correlations are present [10]. If these correlations, which enter the attractive part of the fermionic interaction, are short-ranged $\xi \leq 1$ (in units of the lattice constant) this will favor CDW (at half-filling) competing with s-wave type of pairing. If on the contrary $\xi>1$, the lattice symmetry becomes visible and exotic pairing phases enter the competition [10, 11] along with SDW (at half-filling). This is reflected throughout our results as well as we discuss below.

Our fRG analysis for the fermionic system was applied at zero temperature for the electron-phonon system at half-filling [14]. It involves the self-consistent integration of fast-modes, determined by the energy cutoff $\Lambda(\ell)=4 t_{f} e^{-\ell}$ ( $\ell$ is the RG-step in the integration process) which renormalizes the fermionic interaction until a divergence occurs as the limit $\ell \rightarrow \infty$ is approached. At half-filling the presence of van Hove singularities on the Fermi surface allows us to focus our attention only on $\boldsymbol{k}$-space processes around the singular points $\boldsymbol{Q}_{1}=(\pi, 0)+\boldsymbol{k}$ and $\boldsymbol{Q}_{2}=(0, \pi)+$ $\boldsymbol{k}$ with $|\boldsymbol{k}| \ll \pi$, called the two-patch approximation. As a result, a general fermion-fermion coupling $U_{f f}\left(k_{1}, k_{2}, k_{3}\right)$ reduces in momentum-space to four nonredundant couplings associated with the scattering processes of $g_{1} \equiv U_{f f}\left(\boldsymbol{Q}_{1}, \boldsymbol{Q}_{2}, \boldsymbol{Q}_{2}\right), g_{2} \equiv U_{f f}\left(\boldsymbol{Q}_{1}, \boldsymbol{Q}_{1}, \boldsymbol{Q}_{1}\right)$, $g_{3} \equiv U_{f f}\left(\boldsymbol{Q}_{1}, \boldsymbol{Q}_{1}, \boldsymbol{Q}_{2}\right), g_{4} \equiv U_{f f}\left(\boldsymbol{Q}_{1}, \boldsymbol{Q}_{2}, \boldsymbol{Q}_{1}\right)$, where we have suppressed the frequency dependence for clarity. The different orders of interest are defined in terms

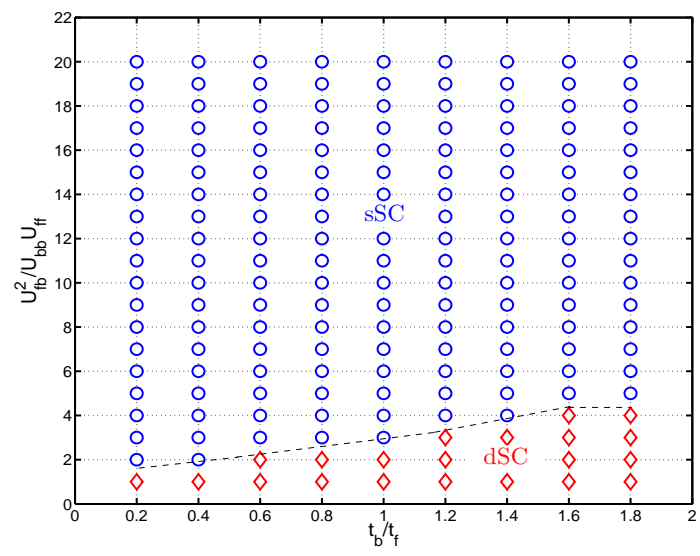

FIG. 2: (Color online) Phase diagram for $U_{f f}=0.4 t_{f}$, $U_{b b}=0.8 t_{f}$, and $n_{b}=2.5$ in the limit $v_{F} \ll v_{s}$ where retardation is not important. In this case, the whole CDW phase disappears. Symbols are same as in Fig. (1) and dashed line is guide to the eye.

of these couplings according to 19

$$
\begin{aligned}
u_{(\mathrm{sd}) \mathrm{SC}} & =-2\left(g_{2} \pm g_{3}\right), \\
u_{\mathrm{SDW}} & =2\left(g_{4}+g_{3}\right), \\
u_{\mathrm{CDW}} & =-2\left(2 g_{1}+g_{3}-g_{4}\right),
\end{aligned}
$$

where the signs are chosen so that all orders diverge to positive values when they strongly renormalize. In this two-patch approximation the effective interaction of Eq. (3) reduces to the pairs of

$$
\begin{aligned}
& g_{1,3}\left(\omega_{1}, \omega_{2}, \omega_{3}\right)=U_{f f}-\frac{U_{f b}^{2}}{U_{b b}} \frac{8 t_{b}^{2} / \xi^{2}}{\left(\omega_{1}-\omega_{3}\right)^{2}+\frac{8 t_{b}^{2}}{\xi^{2}}\left(1+8 \xi^{2}\right)},(7) \\
& g_{2,4}\left(\omega_{1}, \omega_{2}, \omega_{3}\right)=U_{f f}-\frac{U_{f b}^{2}}{U_{b b}} \delta_{\omega_{1}, \omega_{3}},
\end{aligned}
$$

which are inherently anisotropic $\left(\delta_{\omega_{1}, \omega_{3}}\right.$ is the Kronecker delta). This inherent anisotropy can enhance d-wave pairing for example in the regime where $g_{2}<0, g_{3}>0$ with $\left|g_{3}\right|>\left|g_{2}\right|$ as Eq. (4) shows. Finally, in order to account for all frequency channels correctly one needs to evaluate the susceptibilities of the different orders and identify the most divergent one 14 .

We have performed our fRG analysis and have investigated various parameter ranges associated with the experimentally tunable set of $\left\{U_{f f}, U_{f b}, U_{b b}, t_{b}, t_{f}, n_{b}\right\}$. As is reasonably expected, for $U_{f b}^{2} / U_{b b} U_{f f}<1$ the effective interaction of Eq. (3) remains repulsive and antiferromagnetic ordering dominates throughout the range of parameter space. Interesting physics arises when $U_{f b}^{2} / U_{b b} U_{f f} \geq$ 1. In Fig. (11) we present the phase diagram our fRG analysis produces for $U_{f f}=0.4 t_{f}, U_{b b}=0.8 t_{f}$ and $n_{b}=2.5$ and a range of $U_{f b}^{2} / U_{b b} U_{f f}>1, t_{b} / t_{f}$ values. We notice that even at half-filling a large region of the phase diagram is taken over by pairing related phases. This is the essential new result of this work and the difference with 


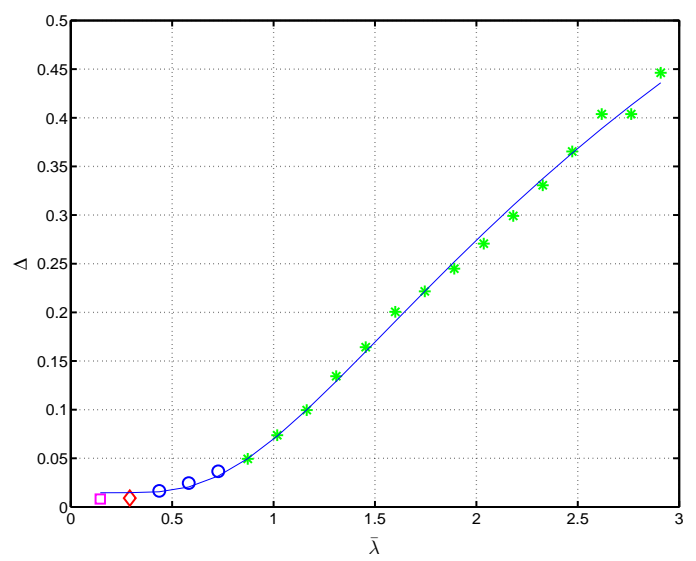

FIG. 3: (Color online) Evolution of the gap along $t_{b} / t_{f}=$ 0.6 of Fig. (1) with identical symbol scheme for the different orders. The blue line fitting was according to $\Delta=0.015+$ $3.326 \exp (-(3.101+\bar{\lambda}) / \bar{\lambda})$.

previous studies, where mean-field[10] and $v_{F}<<v_{s}$ fRG[11] focused on different dopings for the fermionic system. Both were unable to capture at half-filling the rich interplay of orders and their strong dependence on $t_{b}$. As $t_{b}$ is strengthened, the bosonic correlation length $\xi$ (which is independent of $U_{f b}$ ) becomes larger allowing for boson-mediated attraction among the fermions to reach over many lattice sites increasing the Cooper pair sizes and allowing fermions to avoid on-site pairing and the associated energy cost of $U_{f f}$. For small $U_{f b}$ values this mechanism is detrimental for CDW and only when the bosonic correlation length $\xi \leq 1 / 2$ can CDW win over s-wave pairing. For larger values of $U_{f b}$ charge order can survive over sSC in a region $1 / 2 \leq \xi<1$. If the range of the attractive interaction exceeds the lattice constant value then pairing across different sites becomes energetically favorable and CDW becomes subleading.

At this point we should mention that the $\xi \sim 1 / 2$ condition for CDW to appear indicates that the BEC phase has weakened as a whole and long range order has been lost. In free space, the consequence of this is that our linear approximation of coupling fermions to only the bosonic density fluctuations might not be adequate, there will be lots of quasiparticle excitations. Nevertheless, we believe that even if those processes are taken into account the strong influence of the lattice and the presence of nesting on the Fermi surface will sustain the CDW order to be experimentally observed.

As we mentioned in the introduction, all previous works on this type of system have ignored retardation effects. This is an a priori assumption based on comparing the free-space masses of ${ }^{40} \mathrm{~K}$ and ${ }^{87} \mathrm{Rb}[10]$. When the lattice is turned on, one should be comparing band-masses related to $t_{f}, t_{b}$ which can be tuned to any range and consequently $v_{F}, v_{s}$ can be independently set experimentally. In Fig. (2) we show the same phase diagram as in Fig. (1) without retardation taken into consideration, which ef- fectively amounts to setting $\omega_{1}=\omega_{3}$ for all frequency channels in Eqs. (7/8). We have recovered the results at half-filling of the previous fRG study 11]. Antiferromagnetism is expected to take over at the $U_{f b}^{2} / U_{b b} U_{f f}<1$ region. As it can be clearly seen by contrasting the two phase diagrams, retardation is very important at tuning the fermionic phases.

Our analysis has the additional advantage of qualitatively producing the energy gap associated with the dominant orders, $\Delta=4 t_{f} e^{-\ell_{c}}$, just by following the critical RG-step $\left(\ell_{c}\right)$ at which they diverge. We can define

$$
\bar{\lambda}=\frac{U_{f b}^{2}}{2 U_{b b}} \frac{2+8 \xi^{2}}{1+8 \xi^{2}},
$$

as the average anisotropic coupling strength of the fermion-boson interaction (easily derived from Eqs. (77[8]) and investigate the functional form of $\Delta(\bar{\lambda})$. In Fig. (3) we show the evolution of the gap along the $t_{b} / t_{f}=0.6$ direction in Fig. (1), where the fermionic system passes through all four phases. The functional form we use for the fitting is $\Delta=d_{1}+d_{2} \exp \left(-\left(d_{3}+\bar{\lambda}\right) / \bar{\lambda}\right)$ with $d_{1}=0.015, d_{2}=3.326, d_{3}=3.101$.

In conclusion we have applied functional renormalization group method with full retardation for a bosonfermion mixture system on an optical lattice when the fermions are at half-filling and the bosons have BoseEinstein condensed. We find a rich phase diagram providing quantitative estimates for the experimental observation of the competing orders. Additionally, we have produced a functional dependence of the energy gap of the dominant orders with the average boson-fermion coupling strength.

We would like to acknowledge fruitful discussions with Karyn Le Hur and Antonio Castro Neto.

* Electronic address: fkliron@physics.ucr.edu

$\dagger$ Electronic address: swtsai@physics.ucr.edu

[1] M. Greiner et al., Nature 415, 39 (2002)

[2] M. Greiner et al., Nature 426, 537 (2003)

[3] B. Paredes et al., Nature 429, 277 (2004)

[4] Z. Hadzibabic et al., Nature 441, 1118 (2006)

[5] M. Lewenstein et al., cond-mat/0606771

[6] H. P. Büchler and G. Blatter, Phys. Rev. A 69, 063603 (2004); H. P. Büchler and G. Blatter, Phys. Rev. Lett. 91, 130404 (2003)

[7] M. Lewenstein et al., Phys. Rev. Lett. 92, 050401 (2004)

[8] F. Illuminati and A. Albus, Phys. Rev. Lett 93, 090406 (2004)

[9] K. Sengupta, N. Dupuis, and P. Majumdar, cond-mat/0603162

[10] D.-W. Wang, M. D. Lukin, and E. Demler, Phys. Rev. A 72, 051604(R) (2005)

[11] L. Mathey et al., Phys. Rev. Lett. 97, 030601 (2006)

[12] S.-W. Tsai et al., Phys. Rev. B 72, 054531 (2005).

[13] R. Shankar, Rev. Mod. Phys. 66, 129 (1994) 
[14] F. D. Klironomos, S.-W. Tsai, Phys. Rev. B 74, 205109 (2006)

[15] F. Ferlaino et al., Phys. Rev. A 73, 040702(R) (2006)

[16] G. Modugno et al., Science 297, 2240 (2002)
[17] G. Roati et al., Phys. Rev. Lett. 89, 150403 (2002)

[18] C. Ospelkaus et al., Phys. Rev. Lett. 96, 020401 (2006)

[19] H. J. Schulz, Europhys. Lett. 4, 609 (1987) 